\title{
Neurocognitive functioning in acute or early HIV infection
}

\author{
David J. Moore • Scott L. Letendre • Sheldon Morris • \\ Anya Umlauf • Reena Deutsch • Davey M. Smith • \\ Susan Little • Alexandra Rooney • Donald R. Franklin • \\ Ben Gouaux • Shannon LeBlanc • Debra Rosario • \\ Christine Fennema-Notestine $\cdot$ Robert K. Heaton • \\ Ronald J. Ellis • J. Hampton Atkinson • Igor Grant • \\ for the CHARTER Group
}

Received: 07 July 2010 /Revised: 04 November 2010 / Accepted: 08 November 2010 / Published online: 15 December 2010

(C) The Author(s) 2010. This article is published with open access at Springerlink.com

\begin{abstract}
We examined neurocognitive functioning among persons with acute or early HIV infection (AEH) and hypothesized that the neurocognitive performance of AEH individuals would be intermediate between HIV seronegatives (HIV-) and those with chronic HIV infection. Comprehensive neurocognitive testing was accomplished with 39 AEH, 63 chronically HIV infected, and 38 HIVparticipants. All AEH participants were HIV infected for less than 1 year. Average domain deficit scores were
\end{abstract}

D. J. Moore $\cdot$ C. Fennema-Notestine $\cdot$ R. K. Heaton $\cdot$

J. H. Atkinson- I. Grant

Department of Psychiatry, University of California,

San Diego (UCSD),

San Diego, CA, USA

D. J. Moore $(\square) \cdot$ S. L. Letendre $\cdot$ A. Umlauf $\cdot$ R. Deutsch

A. Rooney $\cdot$ D. R. Franklin $\cdot$ B. Gouaux $\cdot$ S. LeBlanc $\cdot$

D. Rosario $\cdot$ C. Fennema-Notestine - R. K. Heaton - R. J. Ellis •

J. H. Atkinson - I. Grant

HIV Neurobehavioral Research Center (HNRC),

University of California, San Diego,

220 Dickinson Street, Suite B, Mail Code 8231, San Diego, CA

92103-8231, USA

e-mail: djmoore@ucsd.edu

S. L. Letendre $\cdot$ S. Morris $\cdot$ D. M. Smith $\cdot$ S. Little Department of Medicine, University of California,

San Diego (UCSD),

San Diego, CA, USA

C. Fennema-Notestine

Department of Radiology, University of California,

San Diego (UCSD),

San Diego, CA, USA

R. J. Ellis

Department of Neurosciences, University of California,

San Diego (UCSD),

San Diego, CA, USA calculated in seven neurocognitive domains. HIV-, AEH, and chronically HIV infected groups were ranked from best (rank of 1) to worst (rank of 3) in each domain. All participants received detailed substance use, neuromedical, and psychiatric evaluations and HIV infected persons provided information on antiretroviral treatment and completed laboratory evaluations including plasma and CSF viral loads. A nonparametric test of ordered alternatives (Page test), and the appropriate nonparametric follow-up test, was used to evaluate level of neuropsychological (NP) functioning across and between groups. The median duration of infection for the AEH group was 16 weeks [interquartile range, IQR: 10.3-40.7] as compared to 4.9 years $[2.8-11.1]$ in the chronic HIV group. A Page test using ranks of average scores in the seven neurocognitive domains showed a significant monotonic trend with the best neurocognitive functioning in the HIV- group (mean rank= 1.43), intermediate neurocognitive functioning in the AEH group (mean rank=1.71), and the worst in the chronically HIV infected (mean rank $=2.86 ; L$ statistic $=94, p<0.01$ ); however, post-hoc testing comparing neurocognitive impairment of each group against each of the other groups showed that the chronically infected group was significantly different from both the HIV- and AEH groups on neurocognitive performance; the AEH group was statistically indistinguishable from the HIV- group. Regression models among HIV infected participants were unable to identify significant predictors of neurocognitive performance. Neurocognitive functioning was worst among persons with chronic HIV infection. Although a significant monotonic trend existed and patterns of the data suggest the AEH individuals may fall intermediate to HIV- and chronic participants, we were not able to statistically confirm this hypothesis. 
Keywords HIV infection $\cdot \mathrm{HIV}$-associated neurocognitive disorders · Acute or early HIV · Primary HIV

\section{Introduction}

Combined antiretroviral therapies (ART) have reduced medical morbidity and mortality due to HIV although neurological complications, especially HIV-associated neurocognitive disorders (HAND), are still common. Severe forms of HAND, such as HIV-associated dementia, are less common in the ART era; however, recent studies found that $30-50 \%$ of HIV-infected persons receiving ART have milder HAND syndromes (Sevigny et al. 2007; Tozzi et al. 2007). The importance of understanding the consequences of HAND is substantial as impairment can be associated with declines in everyday functioning (Heaton et al. 2004b), employment (Heaton et al. 1994), driving (Marcotte et al. 2006), and managing medications (Woods et al. 2009a).

The reasons for the persistence of HAND are not well understood. One possibility is that events during the acute or early stages of HIV infection (AEH; i.e., between 0 - and 52-weeks duration) may set the stage for neurological disease during chronic infection, particularly since AEH is characterized by high levels of HIV replication and organ dissemination (Daar et al. 1991; Little et al. 1999; Schacker et al. 1998), including to the central nervous system (CNS; Tambussi et al. 2000). A preliminary international study suggested that neurocognitive difficulties exist in the early stages of HIV infection including impairments in speed of information processing and verbal fluency (Mandal et al. 2008), and it is recognized that CNS involvement can occur during 'asymptomatic' infection and may precede other disease manifestations (Grant et al. 1987). Neuroimaging studies have also suggested that there are brain alterations early during HIV infection (Lentz et al. 2009) and a recent study by our group suggested that HIV infection may result in early changes to subcortical regions followed by spread to cortical regions (Ances et al. 2009).

To date, there have been no reports comparing AEH and chronically infected persons on comprehensive neurocognitive testing. We examined groups of participants with AEH and chronic infection, as well as HIV negative (HIV-) controls with a standardized battery of neurocognitive tests to ascertain whether there were specific neurocognitive deficits in AEH and to compare such deficits to chronically HIV infected persons. Our working hypothesis was that there would be a trend toward worse neurocognitive performance such that HIV- would manifest the best performance, followed by AEH and then chronically HIV infected. We also explored variables, e.g., viral load, nadir CD4, ART treatment status, which might predict neurocognitive performance.

\section{Methods}

Study population

This study evaluated 39 participants with AEH infection, 63 participants with chronic infection, and 38 individuals who were HIV-. Participants were collected from the UCSD site of the NIH-funded CNS HIV Antiretroviral Therapy Effects Research (CHARTER) study and the UCSD HIV Neurobehavioral Research Center (HNRC). Exclusion criteria included: (1) neurologic disorder not related to HIV infection (e.g., stroke, uncontrolled seizure disorder, head injury with loss of consciousness for longer than $30 \mathrm{~min}$ ), (2) current or past psychotic disorder (e.g., schizophrenia) not related to stimulant or other substance use, (3) conditions that prevent use of consistent neurocognitive testing (e.g., severe visual or hearing impairment), and (4) participants less than 18 years of age. At the time of neurocognitive testing, participants reported no use of illicit substances in the past week (excluding marijuana) and were required to have a negative toxicology screen.

The UCSD Human Research Protection Program approved the current study and all study participants provided written informed consent to participate. At assessment, all subjects completed a blood draw, neuromedical exam, comprehensive neurocognitive testing, detailed substance use history, structured psychiatric interviews for detecting lifetime and current diagnoses of substance use disorders and affective disorders, and a measure of current mood. Laboratory measures included hemogram and routine blood chemistries, plus determination of HIV viral load. Cerebrospinal fluid (CSF) studies (including viral load) were completed with 33/39 AEH and 57/63 chronically infected (see Table 1).

Participants in the AEH group had acute or early HIV infection staged based on the Fiebig classification (Fiebig et al. 2003) and duration of HIV Infection was estimated, as previously described (Volberding et al. 2009), by evaluation of the following data collected at the time of the neurocognitive evaluation, unless otherwise noted: (1) detectable HIV-1 RNA (Amplicor, Roche) and HIV enzyme immunoassay (EIA) negative, or (2), HIV RNA positive and Western Blot indeterminate with no more than three positive bands or alternatively, (3) an HIV RNA positive, EIA positive and detuned EIA consistent with very recent infection of $<133$ days $(\mathrm{OD} \leq 0.75$ by Vironostika, or equivalent by Vitros ECi or OraQuick detuned) (Kothe et al. 2003), or (4) a known last negative EIA, followed by a known positive EIA and neurocognitive assessment within 182 days of the last known EIA negative test.

Participants in the chronic HIV $(\mathrm{CH})$ group were estimated to have HIV infection for more than 1 year at their neurocognitive assessment as defined by the time from 
Table 1 Demographic, substance use, psychiatric, and HIV disease characteristics for the groups

\begin{tabular}{|c|c|c|c|c|c|}
\hline Demographics & $\mathrm{HIV}-(N=38)$ & $\mathrm{AEH}(N=39)$ & Chronic $(N=63)$ & $P$ value & Difference \\
\hline Age, years; mean (SD) & $32.5(9.2)$ & $31.9(8.4)$ & $34.5(5.4)$ & 0.17 & \\
\hline Education, years; mean (SD) & $13.8(2.4)$ & $14.4(2.1)$ & $13.6(2.6)$ & 0.23 & \\
\hline Male & $32(84 \%)$ & $36(92 \%)$ & $58(92 \%)$ & 0.42 & \\
\hline Caucasian & $25(66 \%)$ & $27(69 \%)$ & $37(59 \%)$ & 0.53 & \\
\hline WRAT-III Reading Score, Raw; mean (SD) & $50(5.0)$ & $50(4.1) N=37$ & $49(4.6) N=60$ & 0.68 & \\
\hline Infected with hepatitis $C$ & $2(5 \%)$ & $0(0 \%)$ & $6(10 \%)$ & 0.15 & \\
\hline \multicolumn{6}{|l|}{ Substance abuse/dependence } \\
\hline Alcohol (lifetime) & $14(37 \%)$ & $17(44 \%)$ & $38(60 \%)$ & 0.05 & \\
\hline Marijuana (lifetime) & $13(34 \%)$ & $11(28 \%)$ & $23(37 \%)$ & 0.68 & \\
\hline 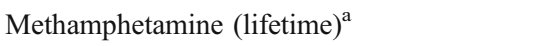 & $16(42 \%)$ & $16(41 \%)$ & $24(38 \%)$ & 0.91 & \\
\hline Cocaine (lifetime) & $5(13 \%)$ & $9(23 \%)$ & $21(33 \%)$ & 0.06 & \\
\hline Opioid (lifetime) & $0(0 \%)$ & $4(10 \%)$ & $7(11 \%)$ & 0.08 & \\
\hline \multicolumn{6}{|l|}{ Psychiatric } \\
\hline Major depressive disorder (lifetime) & $7(18 \%)$ & $19(50 \%) N=38$ & $31(49 \%)$ & 0.003 & $\mathrm{HIV}-<\mathrm{AEH}, \mathrm{CH}$ \\
\hline Major depressive disorder (current) & $2(5 \%)$ & $3(8 \%)$ & $6(10 \%)$ & 0.92 & \\
\hline Beck Depression inventory-II ${ }^{\mathrm{a}}$; mean (SD) & $6.3(8.3)$ & $10.0(8.1)$ & $13.0(10.0)$ & 0.002 & $\mathrm{HIV}-<\mathrm{CH}$ \\
\hline \multicolumn{6}{|l|}{ HIV disease characteristics } \\
\hline CD4 count; mean (SD) & N/A & $538(287)$ & $570(299)$ & 0.60 & \\
\hline Nadir CD4; mean (SD) & N/A & $386(185)$ & $294(225)$ & 0.04 & $\mathrm{AEH}>\mathrm{CH}$ \\
\hline Plasma viral load $\left(\log _{10}\right)^{\mathrm{a}} ;$ median (IQR) & N/A & $4.5(3.1-5.0)$ & $2.3(1.7-4.3)$ & $<0.0001$ & $\mathrm{AEH}>\mathrm{CH}$ \\
\hline Detectable plasma viral load ${ }^{\mathrm{a}}$ & N/A & $37(95 \%)$ & $37(59 \%)$ & $<0.0001$ & $\mathrm{AEH}>\mathrm{CH}$ \\
\hline CSF viral load $\left(\log _{10}\right)$; median (IQR) & N/A & $2.3(1.7-3.4) N=33$ & $1.7(1.7-2.9) N=57$ & 0.11 & \\
\hline Detectable CSF viral load & N/A & $21(64 \%) N=33$ & $22(39 \%) N=57$ & 0.02 & $\mathrm{AEH}>\mathrm{CH}$ \\
\hline Peak plasma viral load $\left(\log _{10}\right)$; median (IQR) & N/A & $5.1(4.8-5.7) N=36$ & $4.9(4.4-5.3) N=55$ & 0.41 & \\
\hline Duration of infection (weeks); median (IQR) & N/A & $16(10-41)$ & $255(144-579)$ & $<0.0001$ & $\mathrm{AEH}<\mathrm{CH}$ \\
\hline ART status (on) $)^{\mathrm{a}}$ & $\mathrm{N} / \mathrm{A}$ & $6(15 \%)$ & $36(57 \%)$ & $<0.0001$ & $\mathrm{AEH}<\mathrm{CH}$ \\
\hline
\end{tabular}

Values are shown as $N(\%)$ unless indicated otherwise

${ }^{a}$ Candidates considered in model selection based on $p<0.10$ criterion for association between variable and Global Deficit Score

the last negative EIA to neurocognitive assessment (with a known positive EIA occurring intermediate to these two time points). For participants using this latter approach (AEH criterion \#4 and chronic HIV criterion), estimated duration infection was calculated from the last known negative test to guard against underestimating duration of HIV infection. The HIV- and chronic HIV participants were selected from a pool of existing participants to be as comparable as possible to the AEH group on demographic (both HIV- and chronic HIV groups), lifetime substance abuse or dependence (both HIV- and chronic HIV groups) and HIV disease (chronic HIV group only) factors (although not possible for all variables) blinded to neurocognitive outcomes.

Neurobehavioral and psychiatric examinations

All participants completed a comprehensive neuropsychological test battery that assessed seven domains (i.e., verbal fluency, executive functioning, speed of information processing, learning, recall, working memory, and motor skills), as previously described (Woods et al. 2004; Heaton et al., in press). Raw test scores were converted to demographically adjusted T-scores using up-to-date normative data (Cherner et al. 2007; Heaton et al. 2004a; Heaton et al. 2003). Tscores were converted into deficit scores ranging from 0 ( $\mathrm{T}$ score $>39$; no impairment) to 5 (T-score $<20$; severe impairment). The deficit scores were then averaged to derive a Global Deficit Score (GDS), which reflected both the number and the severity of deficits across the neuropsychological battery (Carey et al. 2004; Heaton et al. 2004a; Heaton et al. 1995). We also calculated domain deficit scores for each of the seven neurocognitive domains and ranked performance in each of these domains across groups (HIV-, $\mathrm{AEH}$, Chronic) on a simple 1 (best neurocognitive performance) to 3 (worst neurocognitive performance) scale.

Structured mood disorder and substance abuse/dependence diagnoses were obtained using the Composite 
International Diagnostic Interview (Wittchen et al. 1991) and mood symptoms were evaluated with the Beck Depression Inventory-II (Beck et al. 1996). Abuse and dependence were evaluated separately for alcohol, marijuana, cocaine, methamphetamine, and opioid substance groups. To decrease the number of statistical comparisons, participants were collapsed into lifetime and past month abuse/dependence for each of the five substances.

\section{Neuromedical examination}

We performed a comprehensive neuromedical evaluation that included past and current ART use, a structured neurological and medical examination, and collection of blood and urine samples. For those who consented, CSF was collected by lumbar puncture. HCV serostatus was evaluated by standard clinical antibody detection [ADVIA Centaur XP Immunoassay, Siemens Healthcare Diagnostics], and confirmed for active $\mathrm{HCV}$ infection by measuring HCV RNA (Roche Amplicor HCV Monitor Test, nominal detection limit of $28 \mathrm{IU} / \mathrm{mL}$ ).

\section{Statistical analysis}

Descriptive summaries of study groups were reported as mean (SD), median (interquartile range (IQR)), and $N$ (\%). Prior to all statistical testing, the data, either raw or transformed, were reviewed to ensure that test assumptions were valid. Univariable comparisons of demographic, substance abuse/dependence, psychiatric, and neurobehavioral variables among the HIV-, AEH, and chronic HIV groups were performed using ANOVA, Welch's $t$ tests, Chi-square tests, and Fisher Exact tests, depending on the type of data and assumption validity. Among variables found to be significant, pairwise comparison was performed with Tukey's HSD test or logistic regression. The same methods were used to analyze differences in HIV disease characteristics between the $\mathrm{AEH}$ and chronic infection groups.

To test the overall hypothesis that there would be a monotonic trend in neurocognitive data such that performance would be ordered as HIV- (best), AEH (intermediate), and chronically infected (worst), we used the Page test for ordered alternatives. A Page test determined if the medians of mean domain deficit scores monotonically increased in the groups ordered by hypothesized risk (Page 1963). If the Page test produced significant results, pairwise comparisons were performed using a multiple comparison technique adapted from the Friedman two-way ANOVA by ranks test (Siegel and Castellan 1988) that controls the overall 5\% Type I error rate. Tests were one-sided, consistent with our hypothesis, and based on a $5 \%$ significance level.
In addition, to explore how group membership and other variables, including demographic and HIV disease characteristics, were related to neurocognitive functioning, we performed a series of linear regression analyses within HIV+ persons. Included in the analyses were potential confounder variables, defined as univariable predictors of GDS with $p$ values $<0.10$. Spearman rank correlation tests were applied to the continuous potential confounder variables, and those with significant correlation among themselves were not included together in a model. To control for the potential interaction of ART use and viral load, current ART status (on/off) was added to models that included plasma viral load, and their interactions were tested. By process of forward-backward stepwise regression (based on Akaike Information Criterion), each model was reduced to its most simple form (Akaike 1974). We forced the primary variable of interest (AEH vs. $\mathrm{CH}$ ) into all models.

\section{Results}

The sample consisted predominantly of Caucasian men in their early 30 s who had some college education. AEH participants were demographically comparable to chronic HIV and HIV- participants (Table 1). As might be expected, lifetime rates of major depression and substance use disorders were high in HIV+ individuals, while current (1 month) rates of substance use disorders were much lower. There were no significant differences across groups in lifetime rates of substance abuse/dependence, and rates of past month abuse/dependence of other substances were rare and comparable across groups (e.g., the highest rate of abuse/dependence was that $8 \%$ of HIV- group had used marijuana in the past month). In terms of psychiatric characteristics, both HIV+ groups evidenced a significantly higher lifetime prevalence of major depressive disorder (MDD) diagnosis, when compared to the HIV- group, but there were no differences in current MDD rates. The chronic HIV infection group reported significantly higher depressive symptoms scores on the Beck Depression Inventory-II than the HIV- group $(p=0.002)$. Rates of HCV infection were low (0-10\%). In terms of HIV disease characteristics, AEH had an estimated duration of infection of about 4 months as compared to over 4 years for the chronically infected. Whereas the groups were comparable on current CD4 counts, the chronic group reported significantly lower CD4 nadir counts (Table 1). At the same time, plasma viral load was higher in AEH and is consistent with the fact that only $15 \%$ (versus $57 \%$ for the chronically infected) were on ART at time of examination. CSF viral loads were comparable between groups. Proportions of subjects with detectable plasma viral load and $\mathrm{CSF}$ viral load were significantly lower for the $\mathrm{CH}$ group 
(Table 1). Table 1 shows a summary of other HIV disease characteristics for the AEH and chronic HIV groups.

To test the hypothesis that the AEH participants would manifest more neurocognitive difficulties than uninfected controls, but fewer than those with chronic HIV infection, the average domain deficit scores for each of the seven neurocognitive domains were ranked 1 (best group performance) through 3 (worst group performance) in order to apply a Page test for ordered alternatives. The Page test was significant $(p<0.01)$, indicating the presence of a monotonically increasing trend in deficits among the three groups in the order of HIV- (mean rank=1.43), AEH (mean rank= 1.71), and chronically HIV infected (mean $\operatorname{rank}=2.86$; $L$ statistic $=94)$. In follow-on pairwise comparisons, HIVand the $\mathrm{AEH}$ groups were different from the chronically infected group on neurocognitive performance. Although appearing intermediate, the performance of the AEH group did not differ significantly from HIV-.

The results of neurocognitive testing by domain are presented in Fig. 1. The majority of neurocognitive domains (five of seven: executive functioning, speed of information processing, learning, recall, and working memory), as well as global neurocognitive functioning, show a "stair step" pattern where performance is consistent with the hypothesized direction.

A series of linear regressions were performed to model overall level of neurocognitive functioning and determine predictors of performance among $\mathrm{AEH}$ and $\mathrm{CH}$ groups. The variables that were entered into the model are marked by superscript "a" in Table 1 and demographic, substance use, psychiatric, and HIV disease variables were considered. Wide Range Achievement Test, Third Edition (WRAT-III) Reading score was significantly associated with GDS on a univariable basis $(p=0.01)$; however, given that the WRATIII Reading Test is a neuropsychological test, and would be assumed to have some association with overall neurocognitive functioning, we chose to not include this variable in our predictive models.

In a model that included all HIV+ participants, there were no significant predictors of neurocognitive functioning.

\section{Discussion}

In this study, neurocognitive performance was worst among chronically HIV-infected participants as was expected; while the performance of AEH persons trended to fall intermediate between chronically infected and uninfected, we were not able to statistically confirm neurocognitive impairment differences between the $\mathrm{AEH}$ individuals and HIV-uninfected persons. In this particular cohort, no specific HIV disease indicators were associated with the presence of neurocognitive impairment. There are several possible explanations for the lack of an observable intermediate neurocognitive profile for the AEH group.

The first possible explanation is that there are no significant neurobiologic changes early in HIV infection and therefore no neuropsychological impairments. This explanation seems unlikely given that neuroimaging evidence among AEH participants suggests some neurobiologic alterations. Specifically, an arterial spin labeling study reported reductions in regional cerebral blood flow occurred soon after seroconversion; and may possibly reflect neuronal or vascular injury among individuals not yet expressing neurocognitive impairment (Ances et al. 2009). Another recent magnetic resonance spectroscopy study demonstrated neuronal injury or loss (i.e., reduction in $\mathrm{N}$ acetyl aspartate and glutamate+glutamine levels) among AEH participants who presented with seroconversion illness but no neurologic symptoms (Lentz et al. 2009).

The above imaging studies suggest that at least subtle neurobiologic changes are occurring in the earliest phases of HIV infection but that these neurobiologic changes might not be significant enough to produce observable changes in neurocognitive outcomes. Alternatively, it may be that the observable neurocognitive changes are only evidenced during a specific window when HIV is actively disrupting neurobiologic mechanism; as such, subtle neurocognitive changes at this early stage may be transient and difficult to capture at a moment in time.

Finally, it seems equally plausible that the subtle neurobiologic changes result in mild neurocognitive problems
Fig. 1 Means and standard errors of neurocognitive deficit scores for the three groups suggesting that AEH performance falls intermediate to HIV- and chronic HIV groups. The relative ranks within each domain, used in calculating Page test, are listed below each group. Error bars represent standard errors

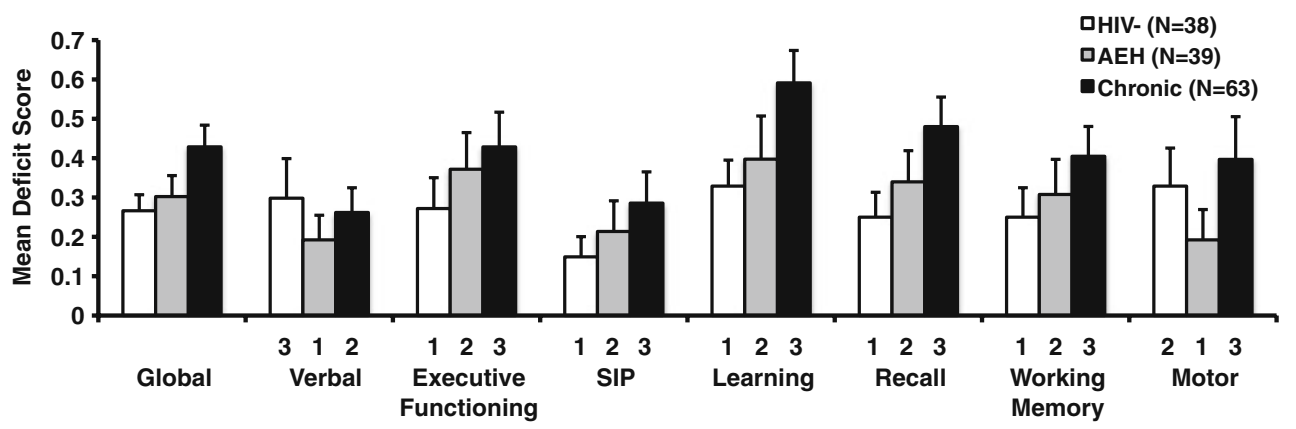


and that these subtle changes are not substantive enough to be statistically captured in a study with a relatively small sample size such as the study we provide here. The non-significant general pattern toward intermediate neurocognitive function for the AEH group (as compared to other two groups) was observed on a global measure, and for five of seven ability domains (Fig. 1), and speaks to this latter possibility. The fact that intermediate indications were most frequently observed in domains that prior research has identified as being particularly sensitive to HIV effects including executive functioning and various aspects of memory (including learning, recall, and working memory) is also supportive of a mild intermediate effect that was not statistically observable here (Reger et al. 2002). The domain of speed of information processing also reflected a tendency toward increasing cognitive difficulties among AEH as compared to HIV- individuals, consistent with previously reported problems in attentional abilities and rapid processing of complex information that have been associated with more advanced HIV disease (Woods et al. 2009b).

The literature has previously suggested some possible mechanisms that may result in the early, subtle changes in neurocognitive performance. The hallmarks of AEH are high levels of HIV replication and wide dissemination of virus, including to the CNS. Replication peaks could lead to neuronal injury by increasing the production of virions and HIV encoded proteins in the CNS. These processes might be facilitated by increasing systemic response and neuroinflammation, which could be accompanied by injury to the blood-brain-barrier. Recent data supporting these possibilities found that worse performance on select neurocognitive measures by AEH participants was associated with higher levels of CSF MCP-1 and IP-10, suggesting monocyte and CD8 T cell activation in the CNS (Peterson et al. 2010).

Our finding that there were no significant predictors of neurocognitive performance in this study speaks to the need to continue to investigate and understand the possible mechanisms responsible for neurocognitive changes among HIV-infected persons. Although the present study is the largest of its kind in terms of comparing $\mathrm{AEH}, \mathrm{CH}$, and HIV- groups with a comprehensive neurocognitive battery, group sizes are still relatively small and the study may not be sufficiently powered to detect predictors of functioning. In order to minimize differences between groups, we matched our $\mathrm{CH}$ group with our AEH group on demographic, substance use, mood, and HIV disease characteristics, and one consequence was that our overall HIV group was primarily male, Caucasian, and had relatively high education levels that may not be representative of the wider HIV-infected population. Therefore, it may be more difficult for subtle NP impairments to be observed in this study as more educated populations may have higher levels of cognitive reserve (Stern 2009). The alternative hypothesis that there is no neurobiological effect strong enough to be neurocognitively detected regardless of cognitive reserve level is also plausible. Importantly, the observation that AEH participants are not statistically different from HIV- persons in NP impairment rates argues against the hypothesis that pre-existing comorbidities are the primary source of impaired NP performance among HIV-infected persons.

It is important that future research follow-up on mechanisms investigated herein with a larger cohort and longitudinal studies (including a neuroimaging component) of persons transitioning through the first weeks and months of HIV infection, and in particular, to link these possible mechanisms to initiation of ART treatment. Another important avenue for future research would be to explore the effects of early initiation of ART with various CNS penetrating capabilities on both neurocognitive outcomes, and potential mediators of neurocognitive change, such as CNS HIV replication, and inflammatory markers.

In summary, neurocognitive functioning is most significantly impacted among the chronically infected. Our study was unable to statistically confirm neurocognitive impairments in the earliest stage of HIV infection, but there is a pattern toward, and non-statistical indicators of, AEH persons showing neurocognitive performance intermediate to HIV- controls and persons with chronic HIV. There are many potential hypotheses for our pattern of results ranging from the lack of a distinct neurobiologic effect to cause neurocognitive changes in the AEH to an inability to detect subtle neurocognitive changes because of power. Because of previous evidence pointing to subtle neurobiologic changes early in disease, we believe that future studies of AEH, particularly those contemplating clinical trials with early ART treatment, should include neurocognitive and neuroimaging measures both to document the potential CNS effects of such interventions and to assess for how they modify neurobiologic and neurocognitive outcomes.

Acknowledgments Supported by NIH contract N01 MH22005 (CHARTER; PI: I. Grant). Although participants in the present study were all taken from the UCSD site, participating CHARTER sites include: Johns Hopkins University (J. McArthur); Mt. Sinai School of Medicine (S. Morgello \& D. Simpson); University of California, San Diego (J.A. McCutchan); University of Texas Medical Branch, Galveston (B. Gelman); University of Washington, Seattle (A. Collier \& C. Marra); Washington University, St. Louis (D. Clifford). Additional support provided by the HIV Neurobehavioral Research Center (HNRC) NIMH Center award MH 62512, NI AI074621 (S. Little, PI), MH083552 (D. Smith, PI), and NIDA Program Project P01DA012065 (I. Grant, PI).

The CNS HIV Antiretroviral Therapy Effects Research (CHARTER) group is affiliated with the Johns Hopkins University, Mount Sinai School of Medicine, University of California, San Diego, University of Texas, Galveston, University of Washington, Seattle, Washington University, St. Louis and is headquartered at the 
University of California, San Diego and includes: Director: Igor Grant, M.D.; Co-Directors: J. Allen McCutchan, M.D., Ronald J. Ellis, M.D., Ph.D., Thomas D. Marcotte, Ph.D.; Center Manager: Donald Franklin, Jr.; Neuromedical Component: Ronald J. Ellis, M.D., Ph.D. (P.I.), J. Allen McCutchan, M.D., Terry Alexander, R.N.; Laboratory, Pharmacology and Immunology Component: Scott Letendre, M.D. (P.I.), Edmund Capparelli, Pharm.D.; Neurobehavioral Component: Robert K. Heaton, Ph.D. (P.I.), J. Hampton Atkinson, M.D., Steven Paul Woods, Psy.D., Matthew Dawson; Virology Component: Joseph K. Wong, M.D. (P.I.); Imaging Component: Christine Fennema-Notestine, Ph.D. (P.I.), Terry L., Jernigan, Ph.D., Michael J. Taylor, Ph.D., Rebecca Theilmann, Ph.D.; Data Management Unit: Anthony C. Gamst, Ph.D. (P. I.), Clint Cushman,; Statistics Unit: Ian Abramson, Ph.D. (P.I.), Florin Vaida, Ph.D.; Protocol Coordinating Component: Thomas D. Marcotte, Ph.D. (P.I.), Rodney von Jaeger, M.P.H.; Johns Hopkins University Site: Justin McArthur (P.I.), Mary Smith; Mount Sinai School of Medicine Site: Susan Morgello, M.D. (Co-P.I.) and David Simpson, M.D. (Co-P.I.), Letty Mintz, N.P.; University of California, San Diego Site: J. Allen McCutchan, M.D. (P.I.), Will Toperoff, N.P..; University of Washington, Seattle Site: Ann Collier, M.D. (Co-P.I.) and Christina Marra, M.D. (Co-P.I.), Trudy Jones, M.N., A.R.N.P.; University of Texas, Galveston Site: Benjamin Gelman, M.D., Ph. D. (P.I.), Eleanor Head, R.N., B.S.N.; and Washington University, St. Louis Site: David Clifford, M.D. (P.I.), Muhammad Al-Lozi, M. D., Mengesha Teshome, M.D.

The views expressed in this article are those of the authors and do not reflect the official policy or position of the Department of the Navy, Department of Defense, nor the US Government.

Open Access This article is distributed under the terms of the Creative Commons Attribution Noncommercial License which permits any noncommercial use, distribution, and reproduction in any medium, provided the original author(s) and source are credited.

\section{References}

Akaike H (1974) New look at the statistical-model identification. IEEE Transactions on Automatic Control Ac 19:716-723

Ances BM, Sisti D, Vaida F, Liang CL, Leontiev O et al (2009) Resting cerebral blood flow: a potential biomarker of the effects of HIV in the brain. Neurology 73:702-708

Beck AT, Steer RA, Brown G (1996) BDI-II Manual. The Psychological Corp, San Antonio

Carey CL, Woods SP, Gonzalez R, Conover E, Marcotte TD et al (2004) Predictive validity of global deficit scores in detecting neuropsychological impairment in HIV infection. J Clin Exp Neuropsychol 26:307-319

Cherner M, Suarez P, Lazzaretto D, Fortuny LA, Mindt MR et al (2007) Demographically corrected norms for the Brief Visuospatial Memory Test-revised and Hopkins Verbal Learning Test-revised in monolingual Spanish speakers from the U. S.-Mexico border region. Arch Clin Neuropsychol 22:343353

Daar ES, Moudgil T, Meyer RD, Ho DD (1991) Transient high levels of viremia in patients with primary human immunodeficiency virus type 1 infection. N Engl J Med 324:961-964

Fiebig EW, Wright DJ, Rawal BD, Garrett PE, Schumacher RT et al (2003) Dynamics of HIV viremia and antibody seroconversion in plasma donors: implications for diagnosis and staging of primary HIV infection. AIDS 17:1871-1879

Grant I, Atkinson JH, Hesselink JR, Kennedy CJ, Richman DD et al (1987) Evidence for early central nervous system involvement in the acquired immunodeficiency syndrome (AIDS) and other human immunodeficiency virus (HIV) infections. Studies with neuropsychologic testing and magnetic resonance imaging. Ann Intern Med 107:828-836

Heaton R, Miller S, Taylor M, Grant I (2004a) Revised comprehensive norms for an expanded Halstead-Reitan battery: demographically adjusted neuropsychological norms for African American and Caucasian adults. Psychological Assessment Resources, Lutz, FL

Heaton, RK, Clifford, DB, Franklin Jr., DR, Woods, SP, Ake, C, et al. HIV-associated neurocognitive disorders persist in the era of potent antiretroviral therapy. Neurology (In press)

Heaton RK, Grant I, Butters N, White DA, Kirson D et al (1995) The HNRC 500-neuropsychology of HIV infection at different disease stages HIV Neurobehavioral Research Center. J Int Neuropsychol Soc 1:231-251

Heaton RK, Marcotte TD, Mindt MR, Sadek J, Moore DJ et al (2004b) The impact of HIV-associated neuropsychological impairment on everyday functioning. J Int Neuropsychol Soc 10:317-331

Heaton RK, Taylor MJ, Manly J (2003) Demographic effects and use of demographically corrected norms with the WAIS-III and WMS-III. In: Tulsky DS, Saklofske DH, Chelune GJ, Heaton RK, Ivnik RJ et al (eds) Clinical Interpretation of the WAIS-III and WMS-III. Academic, San Diego, pp 183-209

Heaton RK, Velin RA, McCutchan JA, Gulevich SJ, Atkinson JH et al (1994) Neuropsychological impairment in human immunodeficiency virus-infection: implications for employment HNRC Group. HIV Neurobehavioral Research Center. Psychosom Med $56: 8-17$

Kothe D, Byers RH, Caudill SP, Satten GA, Janssen RS et al (2003) Performance characteristics of a new less sensitive HIV-1 enzyme immunoassay for use in estimating HIV seroincidence. J Acquir Immune Defic Syndr 33:625-634

Lentz MR, Kim WK, Lee V, Bazner S, Halpern EF et al (2009) Changes in MRS neuronal markers and $\mathrm{T}$ cell phenotypes observed during early HIV infection. Neurology 72:1465-1472

Little SJ, McLean AR, Spina CA, Richman DD, Havlir DV (1999) Viral dynamics of acute HIV-1 infection. J Exp Med 190:841-850

Mandal N, Singh OP, Bhattacharya S, Chatterji S, Biswas A, Sen S (2008) Neurocognitive impairment in early HIV-positive individuals. J Indian Med Assoc 106(442):47-49, p. 53

Marcotte TD, Lazzaretto D, Scott JC, Roberts E, Woods SP, Letendre S (2006) Visual attention deficits are associated with driving accidents in cognitively-impaired HIV-infected individuals. J Clin Exp Neuropsychol 28:13-28

Page EB (1963) Ordered hypotheses for multiple treatments: A significance test for linear ranks. J Am Stat Assoc 58:216-230

Peterson, J, Lee, E, Hecht, F, Price, R, Robertson, K, Spudich, S. 2010. Neurocognitive performance during primary HIV-1 infection. 17th Conference on Retroviruses and Opportunistic Infections, p. Poster 424. San Francisco, California

Reger M, Welsh R, Razani J, Martin DJ, Boone KB (2002) A metaanalysis of the neuropsychological sequelae of HIV infection. J Int Neuropsychol Soc 8:410-424

Schacker TW, Hughes JP, Shea T, Coombs RW, Corey L (1998) Biological and Virologic Characteristics of Primary HIV Infection. Ann Intern Med 128:613-620

Sevigny JJ, Albert SM, McDermott MP, Schifitto G, McArthur JC et al (2007) An evaluation of neurocognitive status and markers of immune activation as predictors of time to death in advanced HIV infection. Arch Neurol 64:97-102

Siegel S, Castellan NJ Jr (1988) Nonparametric statistics for the behavioral sciences. McGraw-Hill, New York

Stern Y (2009) Cognitive reserve. Neuropsychologia 47:2015-2028

Tambussi G, Gori A, Capiluppi B, Balotta C, Papagno L et al (2000) Neurological symptoms during primary human immunodeficiency virus (HIV) infection correlate with high levels of HIV RNA in cerebrospinal fluid. Clin Infect Dis 30:962-965 
Tozzi V, Balestra P, Bellagamba R, Corpolongo A, Salvatori MF et al (2007) Persistence of neuropsychologic deficits despite long-term highly active antiretroviral therapy in patients with HIV-related neurocognitive impairment: prevalence and risk factors. J Acquir Immune Defic Syndr 45:174-182

Volberding P, Demeter L, Bosch RJ, Aga E, Pettinelli C et al (2009) Antiretroviral therapy in acute and recent HIV infection: a prospective multicenter stratified trial of intentionally interrupted treatment. AIDS 23:1987-1995

Wittchen HU, Robins LN, Cottler LB, Sartorius N, Burke JD, Regier D (1991) Cross-cultural feasibility, reliability and sources of variance of the Composite International Diagnostic Interview
(CIDI). The Multicentre WHO/ADAMHA Field Trials. Br J Psychiatry 159(645-53):58

Woods SP, Dawson MS, Weber E, Gibson S, Grant I, Atkinson JH (2009a) Timing is everything: antiretroviral nonadherence is associated with impairment in time-based prospective memory. $\mathrm{J}$ Int Neuropsychol Soc 15:42-52

Woods SP, Moore DJ, Weber E, Grant I (2009b) Cognitive neuropsychology of HIV-associated neurocognitive disorders. Neuropsychol Rev 19:152-168

Woods SP, Rippeth JD, Frol AB, Levy JK, Ryan E et al (2004) Interrater reliability of clinical ratings and neurocognitive diagnoses in HIV. J Clin Exp Neuropsychol 26:759-778 